\title{
Spin Fine Structure in Optically Excited Quantum Dot Molecules
}

\author{
M. Scheibner ${ }^{1}$ * M. F. Doty ${ }^{1}$, I. V. Ponomarev ${ }^{1}$, A.S. Bracker ${ }^{1}$, \\ E.A. Stinaff ${ }^{1}$, V.L. Korenev ${ }^{2}$, T.L. Reinecke ${ }^{1}$, and D. Gammon ${ }^{1}$ \\ ${ }^{1}$ Naval Research Laboratory, Washington, DC 20375, USA and \\ ${ }^{2}$ A.F. Ioffe Physical Technical Institute, St. Petersburg 194021 Russia
}

(Dated: September 8, 2018)

\begin{abstract}
The interaction between spins in coupled quantum dots is revealed in distinct fine structure patterns in the measured optical spectra of InAs/GaAs double quantum dot molecules containing zero, one, or two excess holes. The fine structure is explained well in terms of a uniquely molecular interplay of spin exchange interactions, Pauli exclusion and orbital tunneling. This knowledge is critical for converting quantum dot molecule tunneling into a means of optically coupling not just orbitals but also spins.
\end{abstract}

PACS numbers: 78.67.Hc, 73.21.La, 78.47.+p, 78.55.Et

\section{INTRODUCTION}

Exchange coupling between spins in a double quantum dot molecule (QDM) is an essential component for spin-based quantum information ${ }^{1,2,3}$. Rapid progress has been made recently in doubly charged QDMs that are measured and controlled electrically ${ }^{4,5}$. A corresponding understanding of the spin-spin interactions in optically controlled QDMs is not yet available. These systems could lead to ultrafast, wireless control of spin qubits and optical entanglement of two spins in two dots. To this end it is now critical to obtain a detailed measurement and understanding of the spin states of optically excited QDMs.

In optically excited quantum dots (QDs), an electronhole $(e-h)$ pair is created in the presence of the previously existing $\operatorname{spin}(\mathrm{s})$. As we will show, the resulting $e-e, h-h$, and $e-h$ exchange interactions determine the spin states and can be directly measured through fine structure in the spectra ${ }^{6.7}$. Moreover, electron or hole levels may be coupled by carrier tunneling in a QDM, with the orbital wavefunctions continuously tuneable from atomic to molecular in nature. Recently, photoluminescence (PL) spectra of vertically-stacked InAs/GaAs QDMs measured as a function of electric field have led to the clear identification of tunnel coupling in neutral ${ }^{8,9,10,11,12,13}$ and charged QDMs. ${ }^{10,12,14,15}$ In these earlier studies some limited information on the spin fine structure can be found. Here we give the broad picture of the spin fine structure physics in QDMs

Of great importance is the case of a doubly charged QDM because of its potential use as a two qubit system. To understand this system, we examine cases of zero, one and two charges in the QDM. We demonstrate that all of the observed fine structure features of these systems arise from combinations of three fundamental quantum mechanical processes - tunneling, exchange, and Pauli exclusion. An ideal tool to visualize this underlying physics are PL spectra as function of electric field (see Fig. 1). Such two dimensional color scale plots reveal clearly a

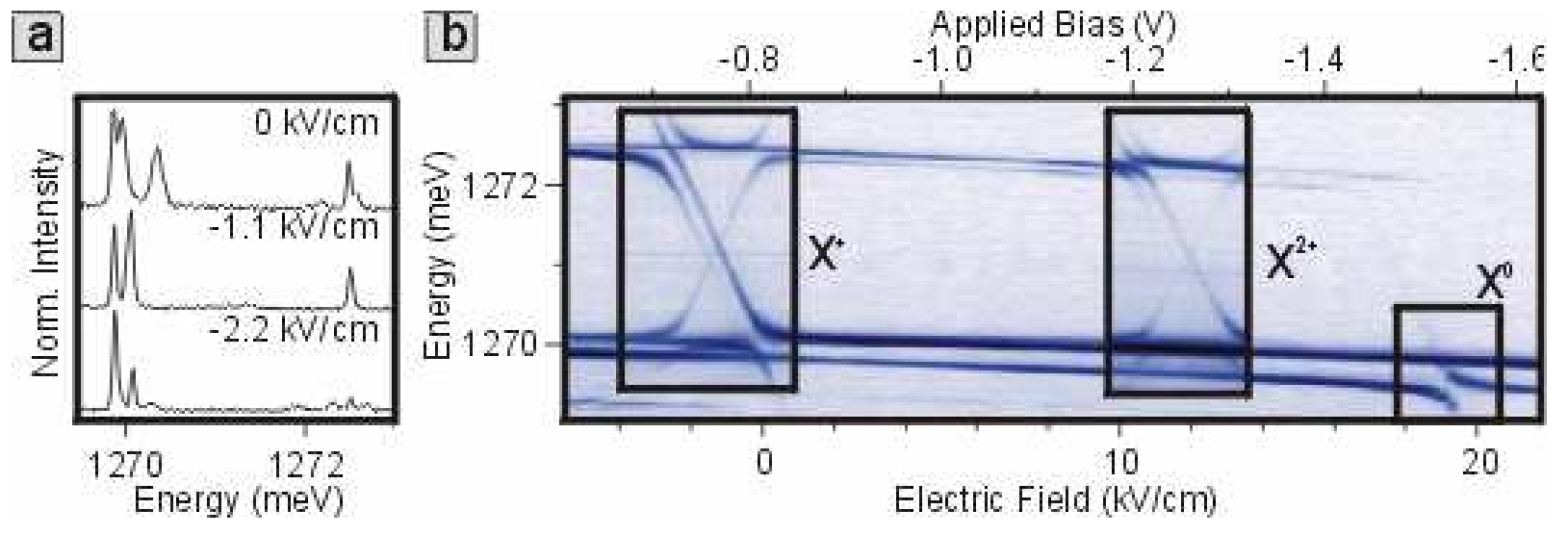

FIG. 1: (Color online) (a) Conventional PL line spectra at selected electric fields. (b) Fine structure of molecular resonances in the PL transitions of the neutral exciton $\left(X^{0}\right)$, positive trion $\left(X^{+}\right)$and doubly positively charged exciton $\left(X^{2+}\right)$ in a QDM is revealed clearly by a 2-dimensional intensity color scale plot of the PL signal. Here the bottom dot has a vertical height of $h_{B}=4 \mathrm{~nm}$ and the top dot has a vertical height of $h_{T}=2.5 \mathrm{~nm}$ with a dot separation of $d=4 \mathrm{~nm}$. 
rich variety of fine structure in the PL transitions of the different excitonic charge states. The primary goal of this paper is to explain the origin of the various fine structure patterns observed at the anticrossing points in such spectra.

\section{SYNOPSIS}

Here we study spin-spin interactions of (pseudo-)spin$1 / 2$ particles, namely electrons and holes, in the vicinity of molecular resonances in double-dot QDMs. Four fundamental cases can be identified. These are depicted in Fig. 2,

The first and simplest case (Fig. 2(a)) is the molecular resonance of a single spin-carrying particle - in this example, a hole. In the absence of a magnetic field, all states are two-fold degenerate. The hole can occupy either of the two QDs, as indicated in the $\boxplus$ diagrams. The potential energies of the two configurations change relative to each other with electric field, because the two QDs are located at a slightly different position within an applied electric field. Without loss of generality we chose the energy of the configuration with the hole in the bottom QD to be field independent. As the energies of the two configurations approach each other, the hole wavefunction begins to form bonding (lower energy) and anti-bonding (higher energy) orbital states between the two QDs. In the field dependent energy diagram, Fig. 2(a), this results in an avoided crossing of the energy levels of the two charge configurations. The energy splitting between the bonding and antibonding states is twice the tunnel coupling constant $(\Delta=2 t)$. In the experiment this case is realized by a QDM which contains a single hole. It is observed as the final state in the optical recombination of a positively charged exciton (trion).

The behavior which results from adding another spin depends on the nature of the spin carrying particle. If an electron and a hole occupy the QDM, the physics of the fine structure splitting across a molecular resonance of one of the two particles is as depicted in Fig. 2(b). $e-h$ exchange interaction $\left(J^{e h}\right)$ leads to a small splitting between the states with parallel and antiparallel spins. With both spins in the same QD the interaction between the spins is maximum. The splitting reduces across the molecular resonance as one spin tunnels into the second QD. For both spin configurations (parallel and antiparallel) the splitting between bonding and anti-bonding states is twice the tunneling constant $(\Delta=2 t)$. Examples for this type of behavior are the neutral exciton and the doubly charged exciton. There the spin-spin interaction is characterized by the $e-h$ exchange energy $J^{e h}$.

Figure 2(c) captures the physics in the third case, where both particles are of the same type, e.g. two holes, and may share the same orbital state. At a molecular resonance the Pauli principle demands that tunneling into the same QD can only occur if the particles form a spin-singlet. The three (degenerate) spin-triplet states are unaffected by a molecular resonance. Consequently, tunneling splits singlet from triplet states (kinetic exchange). Note, only one of three degenerate triplet states is depicted in Fig. 2(c). The anticrossing splitting of the singlet states amounts to $\Delta=2 \sqrt{2} t$. This is larger by a factor of $\sqrt{2}$ than for a single particle and

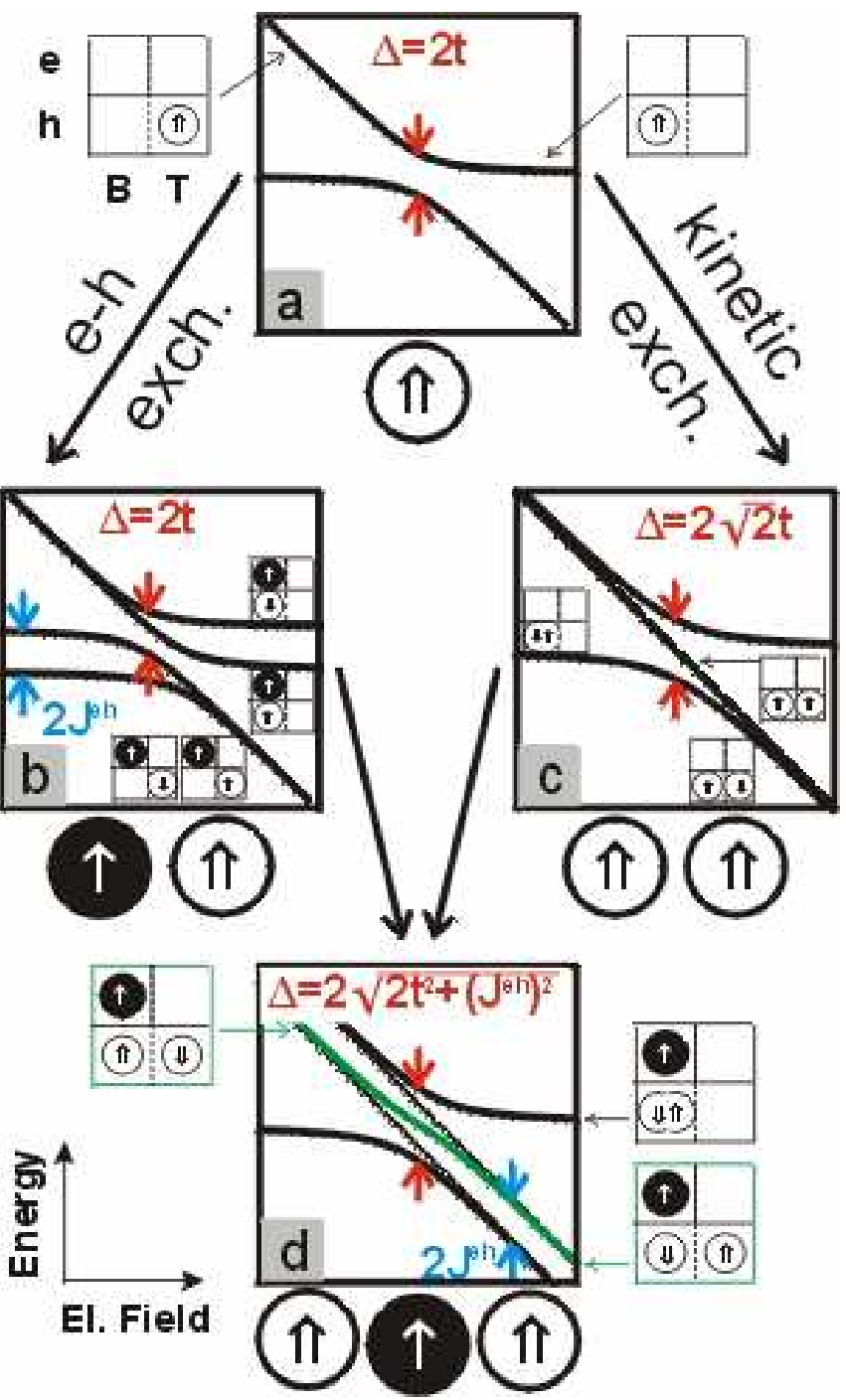

FIG. 2: (Color online) Schematic energy dispersions diagrams (state energy vs. electric field) for a QD molecular resonance of spin carrying particles in two spatial configurations of: (a) A single (spin carrying) particle, (b) Two spin carrying particles of different type (e.g., electron and hole), where only one of them tunnels, (c) Two holes, where both may share the same orbital state, and (d) Three spin carrying particles, e.g. two holes and one electron which does not take part in the tunneling process. In the $\boxplus$ the two left squares represent the bottom QD's and the two right squares the top QD's valence and conduction band. The •'s and o's denote electrons and holes respectively. 
results from the indistinguishability of the two particles. Physical implementations of this case are, for example, QDMs occupied by two holes, or two electrons, or a neutral biexciton with either the two electrons or the two holes spin paired and restricted to one of the QDs.

The fourth case combines the previous two cases (Fig. 2(d)). It is obtained by considering, for example, two holes and one electron, where the holes may tunnel but the location of the electron is fixed to the bottom QD. Here the $e-h$ exchange interaction lifts the degeneracy of the three hole spin triplet states in the region of the molecular resonance. While the two triplet states with parallel spins remain unaffected by the resonance (dashed lines), the third triplet state couples now to the singlet states and 'wiggles' through the resonance region (green line). The total anticrossing splitting in this case amounts to $\Delta=2 \sqrt{2 t^{2}+2\left(J^{e h}\right)^{2}}$. By following the green line in Fig. 2(d) through the resonance it is found that the two holes swap their spin. This exemplifies that the interplay of tunneling and spin interactions can in principle be used to manipulate spin states. The singly charged excitons (trions) are examples where such a situation is realized experimentally.

In the following we will give the details of the experimental observation of these spin fine structure patterns. In particular we will examine the neutral exciton, $X^{0}$, and the doubly positively charged exciton, $X^{2+}$, which illustrate $e-h$ exchange in the presence of hole tunneling. The optical transition of the $X^{2+}$ leaves the QDM in a 2-hole state and thus also shows the kinetic $h-h$ exchange splitting, which is created by tunneling and Pauli exclusion. For the singly charged exciton, $X^{+}$, we find that the interplay of all these processes results indeed in the 'wiggling' of a spectral line, which is a signature of the mixing of spin singlet and spin triplet states. Note, although all experiments are performed with hole tunneling, we find in other experiments that electron tunneling yields qualitatively the same physics.

\section{SAMPLES AND METHODS}

We form QDMs by the subsequent growth of two closely spaced layers of self assembled InAs/GaAs QDs. Because of strain the QDs of the second layer nucleate preferentially on top of QDs in the first layer. The QDMs are embedded in the insulating region of an n-I Schottky diode grown by molecular beam epitaxy on top of a (100) n-type GaAs substrate wafer. The layer sequence is depicted in Fig. 3(a). In order to produce a gradient of the QD density across the wafer the rotation of the sample during growth was halted for the formation of the QD layers. This ensures that we obtain the right density for studying individual molecules in each sample growth. The electric contacts on top of the samples were formed by a semi transparent titanium layer and an aluminum mask with $1 \mu \mathrm{m}$ size apertures for the optical access. We control the heights of the QDs by an indium flush technique. The self-assembled QDs are partially capped with GaAs followed by an increase in temperature in order to redistribute and partly remove the still exposed part of the QD material. For the QDMs discussed here the nominal heights of the bottom dot, $h_{B}$, and the top dot, $h_{T}$, were chosen so that predominantly the bottom dot exhibits the lower transition energy, i.e., $h_{T} \leq h_{B} \frac{13}{}$. This allows the hole levels to be brought into resonance with a positive electric field, while the electron level of the top dot $(T)$ is shifted to much higher energies relative to that of the bottom $\operatorname{dot}(B)$ (see Fig. 3(b)). In this case, we include in our discussion only the "atomic" s-shell orbital states for the electron localized in the bottom dot and for the holes in both dots. We consider samples with relatively wide interdot barriers $(d \geq 4 \mathrm{~nm})$ such that hole tunneling rates are small $(\leq 1 \mathrm{meV}) \underline{\underline{13}}$

The case of electron tunneling is realized using the same principle. It is achieved by either a reversed dot order $\underline{8}$ or by applying a negative electric field $\stackrel{13}{ }$ In the latter case a p-type instead of an n-type substrate is required for the proper pinning of the Fermi level in the Schottky diode.

Apertures in the aluminum mask with a diameter of about $1 \mu \mathrm{m}$ allow us to address and detect individual QDMs optically. The QDMs were excited below the energy of the wetting layer with a frequency tunable continuous wave titanium sapphire laser. A liquid nitrogen cooled charge coupled device (CCD) camera in combination with a $1200 \mathrm{~mm}^{-1}$ line grating inside a $0.55 \mathrm{~m}$ monochromator provided an overall spectral resolution of $50 \mu \mathrm{eV}$ for the detection of the PL. The samples were measured at a temperature of $10 \mathrm{~K}$. The interplay of optical excitation, recombination, and tunneling to and from the substrate leads to the observation of several charge states in the same PL spectrum. ${ }^{16,17}$
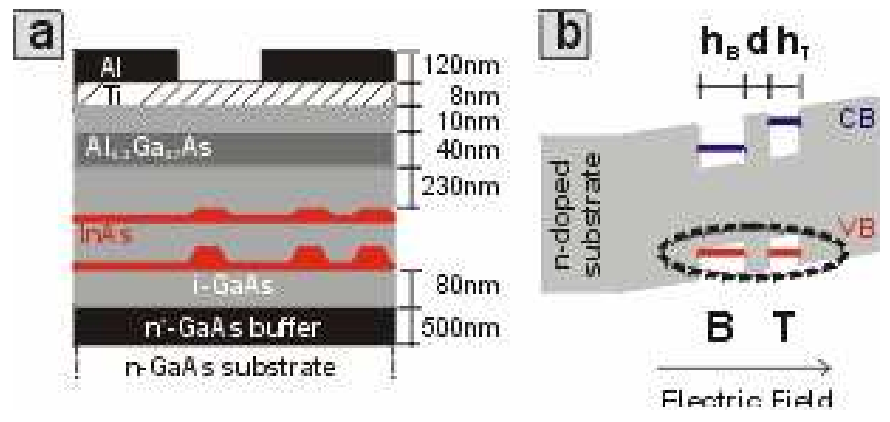

FIG. 3: (Color online) (a) Schematic of the sample layer sequence. The $\mathrm{n}^{+}$-GaAs buffer layer was doped with Tellurium at $\approx 10^{15} \mathrm{~cm}^{-3}$. (b) Schematic of the QDM diode level structure. 


\section{NOMENCLATURE}

To describe the quantum states of a QDM we use $e_{B} e_{T}$
$h_{B} h_{T}$$X^{q}$, where $e_{B(T)}$ and $h_{B(T)}$ are the number of electrons and holes in the respective dot and $q$ is the total charge. For example, ${ }_{21}^{10} X^{2+}$ for a doubly positively charged exciton, i.e., 1 electron and 2 holes in the bottom dot and 0 electron and 1 hole in the top dot. Likewise ${ }_{21}^{10} X^{2+}$ corresponds to the interdot transition, ${ }_{21}^{10} X^{2+} \Rightarrow$ ${ }_{20}^{00} h^{2+}$, where $h^{2+}$ is a 2-hole state. We label specific spin states of a charge configuration as, e.g., $\uparrow_{\uparrow, \Downarrow, 0}^{\uparrow} X^{2+}$, where we use the fact that a hole in the ground state of a QD can take only two spin projections $(\Uparrow, \Downarrow \equiv \pm 3 / 2)$, similar to the case of the spin- $1 / 2$ electron $(\uparrow, \downarrow)^{18}$. We specify the hole spin singlet and triplet configurations as, e.g., ${ }_{\Uparrow, \Downarrow,}^{\uparrow, 0} X_{S}^{+}$and ${ }_{\Uparrow, \Downarrow}^{\uparrow, 0} X_{T}^{+19}$. These various state configurations correspond to an "atomic"-like basis in which the spatial wavefunctions are localized predominantly in one or the other QD. They are realized away from the molecular resonances. In the resonance regions the "atomic" states are mixed by tunneling into "molecular" states. The electrons and holes are treated as non-identical particles with an explicit exchange interaction between them.

\section{THEORETICAL DESCRIPTION}

The few-particle Hamiltonians that describe these excitonic states consist of three parts. The first is a sum of electron and hole single-particle QD Hamiltonians $\widehat{\mathbf{h}}^{e(h)}$, the second describes Coulomb interactions, and the third is a short-range electron-hole $(e-h)$ exchange: $A \sum_{i, j} \delta\left(\mathbf{r}_{e i}-\mathbf{r}_{h j}\right) \hat{\sigma}_{z i}^{e} \hat{\sigma}_{z j}^{h}$, where $A$ is the exchange amplitude. We use three s-shell orbitals: the bottom dot electron and hole states and the top dot hole or electron state. The fourth s-shell orbital is tuned far out of resonance and can therefore be neglected.

The Coulomb terms are:

$$
V_{i j k l}^{\alpha, \beta}= \pm \int d \mathbf{r} d \mathbf{r}^{\prime}\left|\mathbf{r}-\mathbf{r}^{\prime}\right|^{-1} \varphi_{i}^{\alpha *}(\mathbf{r}) \varphi_{k}^{\beta *}\left(\mathbf{r}^{\prime}\right) \varphi_{j}^{\alpha}(\mathbf{r}) \varphi_{l}^{\beta}\left(\mathbf{r}^{\prime}\right) \text {, }
$$

with the orthonormalized wavefunction $\varphi_{i}^{\alpha}$ of particle $\alpha$ in $\operatorname{dot} i$. In the wide barrier limit only the direct Coulomb interactions, e.g. the repulsion of two holes in the bottom dot $V_{B B}^{h h} \equiv V_{B B B B}^{h h}$, or between the two dots, $V_{B T}^{h h} \equiv V_{B B T T}^{h h}$ are non-negligible. In addition only $e-h$ exchange within the bottom dot with $J^{e h} \equiv J_{B B}^{e h}=$ $A \int d \mathbf{r}\left|\varphi_{B}^{e}\right|^{2}\left|\varphi_{B}^{h}\right|^{2}$ has to be considered. A theoretical discussion of the general case, including the narrow barrier limit, will be given elsewhere ${ }^{20}$

In the following the bottom QD is chosen as an energy reference point. This means, that the energies of configurations with no charges in the top QD are independent of the applied electric field, $F$, while those with one hole (electron) in the top dot change with field proportional to $f \equiv p F=|e|\left(d+\left(h_{B}+h_{T}\right) / 2\right) F$, and those with 2 holes (electrons) change proportional to $2 f$. Here $e$ is the elementary charge, $d$ is the separation of the dots and $h_{B}$, $h_{T}$ are the heights of the dots. Thus, $p$ is equivalent to the dipole moment of two elementary charges separated by $d$. It follows that intradot transitions, which keep the number of holes (electrons) in the top QD constant, have field-independent PL lines, while interdot transitions have slope $\pm p$.

Nine parameters are physically relevant - the six Coulomb interactions $V_{B B}^{h h}, \quad V_{B B}^{e h}, V_{B B}^{e e}, V_{B T}^{h h}\left(V_{B T}^{e e}\right)$, $V_{B T}^{e h}$ and $V_{T T}^{h h}\left(V_{T T}^{e e}\right)$, the intradot $e-h$ exchange interaction, $J_{B B}^{e h}$, the tunneling matrix element $t_{h(e)}=$ $-\left\langle\varphi_{B}^{h(e)}\left|\widehat{\mathbf{h}}^{h(e)}\right| \varphi_{T}^{h(e)}\right\rangle$, and the dipole moment $p$. Maximally four parameters are required in order to fit the spectral patterns, which are obtained by dispersing the optical transition spectrum of a given excitonic charge state in electric field. These parameters are an effective Coulomb potential, which is given by a combination of the above Coulomb terms, the $e-h$ exchange, the tunneling rate and the dipole moment.

In the course of this paper we will give the Hamiltonians of the respective charge states in matrix representation. For example, a single hole in a QDM represented by the two basis states $|1\rangle={ }_{10}^{00} h^{+}$and $|2\rangle={ }_{01}^{00} h^{+}$is described by:

$$
H^{h} \equiv\left(\begin{array}{ll}
H_{11} & H_{12} \\
H_{21} & H_{22}
\end{array}\right)=\left(\begin{array}{cc}
0 & t_{h} \\
t_{h} & -f
\end{array}\right)
$$

For the multi particle cases we will choose mainly basis states, $|i\rangle$, with diagonal matrix elements, $H_{i i}$, that contain the field dependence, $e-h$ exchange, and some combination of the above Coulomb potentials specific to the respective charge configuration. These can be associated easily with the (atomic) energy levels away from the molecular resonances. In this case coupling between the basis states $|i\rangle$ and $|j\rangle$ will be represented by nonzero off-diagonal matrix elements $\left(H_{i j}\right.$ with $\left.i \neq j\right)$ proportional to $t_{h(e)}$. The off-diagonal "tunneling" terms in the Hamiltonian lead to the formation of molecular states.

This theoretical description yields qualitatively the same results for the two cases of electron and hole tunneling. Both cases differ only in the magnitude of the respective matrix elements and the sign of the field dependence. In our experiments we have so far not found any indication for a qualitative difference between electron and hole tunneling. Because the tunneling rates are larger ${ }^{13}$ and PL transitions overlap more strongly in the case of electron tunneling, we will focus in the following on the case of hole level resonances in order to give a clear picture of the underlying physics. 


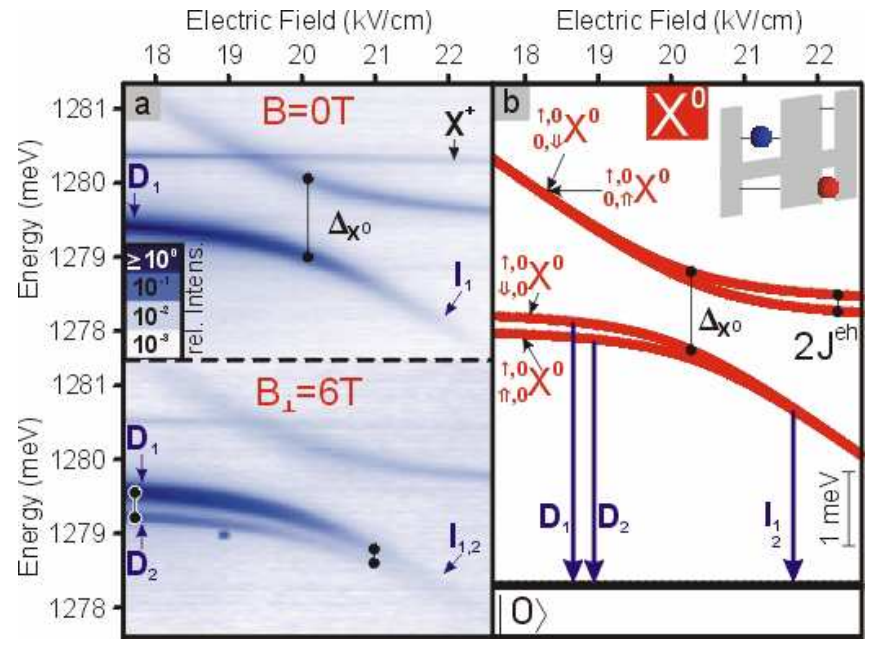

FIG. 4: (Color online) Exciton $\left(X^{0}\right)$ in an uncharged QDM $\left(h_{B}=h_{T}=2.5 \mathrm{~nm}, d=4 \mathrm{~nm}\right)$. (a) The PL spectrum as a function of electric field - at zero magnetic field $(B=0)$ (top) and in a transverse magnetic field $(B=6 \mathrm{~T})$ (bottom) (b) The calculated energy diagram $\left(\Delta_{X^{0}}=2 t_{h}=1.05 \mathrm{meV}\right.$, $\left.2 J^{e h}=240 \mu \mathrm{eV}\right)$. The direct exciton and its corresponding transition $\left(D_{1,2} \equiv \frac{10}{10}^{0} X^{0}\right)$ has the hole in the same dot as the electron and its energy is almost independent of electric field, while the indirect exciton $\left(I_{1,2} \equiv \frac{10}{01}^{0} X^{0}\right)$ has the hole in the opposite dot and its energy changes linearly with field. An anticrossing occurs when their energies are resonant.

\section{ELECTRON-HOLE EXCHANGE}

\section{A. The neutral exciton}

We consider first the case of $e$ - $h$ exchange for the neutral exciton, $X^{0} \equiv 1$ electron +1 hole, in a QDM (Fig. 4). The exciton has spin states that are optically allowed (bright, $\uparrow \Downarrow$ and $\downarrow \uparrow$ ), or optically forbidden (dark, $\uparrow \uparrow$ and $\downarrow \Downarrow)$ 21,22 In what follows we will not list spin degenerate states in which all spins, including the electron spin, are flipped. At zero magnetic field a single intradot exciton line, $\frac{10}{10} X^{0}$, anticrosses with the relatively weak interdot transition, $\underline{10}_{0}^{0} X^{0}$, with a splitting of $\Delta_{X^{0}}=1.0 \mathrm{meV}$ (Fig. 4(a) top). When a transverse magnetic field of $B=6 \mathrm{~T}$ is applied (Voigt geometry) a second, normally dark, intradot spectral line appears $320 \mu \mathrm{eV}$ lower in energy (Fig 4(a) bottom), because the transverse magnetic field mixes the bright and dark states 23 . The bright-dark splitting of the intradot exciton, ${ }_{10}^{10} X^{0}$, arises from e-h exchange, similar to the case of a single dot. As the intradot exciton evolves into the interdot exciton through the anticrossing region, the $e-h$ exchange splitting decreases substantially because of the decreased overlap of the electron and hole wavefunctions in the interdot configuration, ${ }_{01}^{10} X^{0}$.

This physics is captured by calculations of the energy level structure as seen in Fig. 4(b), where we have included $e-h$ exchange, $J^{e h}$, only for the intradot configu- rations ${ }_{10}^{10} X^{0}$. Here the Hamiltonian of the bright $(|1\rangle=$ $\left.{ }_{\Downarrow 0}^{\uparrow 0} X^{0},|2\rangle={ }_{0 \Downarrow}^{\uparrow 0} X^{0}\right)$ and dark $\left(|3\rangle={ }_{\Uparrow 0}^{\uparrow 0} X^{0},|4\rangle={ }_{0 \Uparrow 0}^{\uparrow 0} X^{0}\right)$ excitons at zero magnetic field are

$$
\widehat{H}^{X^{0}}=\left(\begin{array}{cccc}
J^{e h} & t_{h} & 0 & 0 \\
t_{h} & -f & 0 & 0 \\
0 & 0 & -J^{e h} & t_{h} \\
0 & 0 & t_{h} & -f
\end{array}\right)
$$

where energy and field are relative to the center of the exciton anticrossing and $t_{h}$ is the single hole tunneling rate $^{24}$. The applied electric field, $F$, changes the hole energy in the top QD relative to that in the bottom QD by $-f$. The neutral exciton exemplifies that in a QDM $e-h$ exchange is large within the same dot but small between dots.

\section{B. The doubly charged exciton}

When we have two or more electrons (or holes) we must also consider $e-e$ (or $h$ - $h$ ) interactions. The case of two electrons has been discussed for dots controlled by electrical gating. ${ }^{4,5,25}$ The case of two holes is qualitatively the same and is observed optically in the doubly positively charged exciton $\left(X^{2+}\right)$. In contrast to the uncharged exciton, the doubly charged exciton transition shows an ' $\mathrm{x}$ '-pattern ${ }^{10}$ with four anticrossings that involve two direct $(A$ and $D)$ and two indirect ( $B$ and $C$ ) transitions, as seen in Fig. [5(a). This transition pattern is understood using the calculated energy diagrams for the initial $X^{2+}$ states and the final 2-hole states left after recombination, which both have hole level resonances (Fig $5(\mathrm{~b}))$.

Each anticrossing has fine structure whose pattern provides a signature of the corresponding spin configurations. The two anticrossings on the left in the spectrum of Fig. [5) (a) arise from the doubly charged exciton states $\left(\Delta_{X^{2+}}\right)$ and those on the right from the 2-hole states $\left(\Delta_{h^{2+}}\right)$. We first consider the initial states of the $X^{2+}$ transitions, as seen at the top of Fig. 5(b). With respect to spin, the $X^{2+}$ states are qualitatively the same as the states of the neutral exciton. In particular, $e-h$ exchange is present only when the electron shares the bottom dot with the unpaired hole, ${ }_{12}^{10} X^{2+}$. The $e-h$ exchange splitting decreases as the bottom QD is filled with two spin paired holes (total spin zero) and the top QD is left with an unpaired hole, ${ }_{21}^{10} X^{2+}$. Note that this is visible in the $X^{2+}$ transitions even without magnetic field (see Fig. 5)(a) top left corner).

This is described by the Hamiltonian for the basis

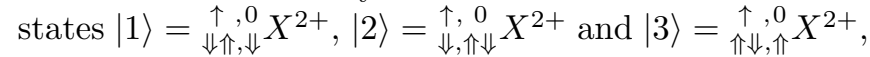




$$
\begin{aligned}
& |4\rangle=\underset{\uparrow, \Downarrow \Uparrow}{\uparrow, 0} X^{2+}, \\
& \widehat{H}^{X^{2+}}=\left(\begin{array}{cccc}
-f & t_{h} & 0 & 0 \\
t_{h} & E-2 f+J^{e h} & 0 & 0 \\
0 & 0 & -f & t_{h} \\
0 & 0 & t_{h} & E-2 f-J^{e h}
\end{array}\right),
\end{aligned}
$$

where $E=V_{B B}^{e h}-V_{B T}^{e h}+V_{T T}^{h h}-V_{B B}^{h h}$. Note, that this is similar to the Hamiltonian of the neutral exciton.

\section{VII. 'KINETIC' EXCHANGE: TWO-HOLE STATES}

If for the final state both holes are in the same dot $\left({ }_{02}^{00} h^{2+}\right)$ the Pauli principle requires that there can be only a spin singlet state. On the other hand, if the holes are each in a different dot $\left({ }_{11}^{00} h^{2+}\right)$ there will be a singlet and three triplet states $\stackrel{27}{ }$ For large barrier width $(d \geq 4 \mathrm{~nm})$ the interdot $h$ - $h$ exchange is negligible, and the spin singlet and triplet states are degenerate at electric fields away from the anticrossing region. However, because tunneling conserves spin, only the interdot singlet configuration ${ }_{\Uparrow, \Downarrow}^{0,0} h_{S}^{2+}$ mixes with the intradot singlet ${ }_{0, \Uparrow \Downarrow}^{0,0} h_{S}^{2+}$, and the degenerate triplet states ${ }_{11}^{00} h_{T}^{2+}$ pass through unaffected as shown in Fig 5 (b). Mixing of the singlet states in the anticrossing region leads to an effective or "kinetic" 28 - $h$ - $h$ exchange splitting between the singlet and the three degenerate triplets, even though interdot $h$ - $h$ exchange is negligible for wide barriers. This type of exchange, which is highly sensitive to the applied electric field through the resonant tunneling, provides a basis for externally manipulating the spin coupling. $\underline{\underline{4}}$

The full Hamiltonian of two holes in a QDM is given in appendix $\mathrm{B}$. The reduced Hamiltonian for the two singlet states, $|1\rangle={ }_{02}^{00} h_{S}^{2+}$ and $|2\rangle={ }_{11}^{00} h_{S}^{2+}$, that are relevant here is

$$
\widehat{H}_{S}^{h^{2+}}=\left(\begin{array}{cc}
V_{T T}^{h h}-2 f & \sqrt{2} t_{h} \\
\sqrt{2} t_{h} & V_{B T}^{h h}-f
\end{array}\right),
$$

The factor of $\sqrt{2}$ in the tunneling rate between the singlet states comes from the fact that two indistinguishable holes can tunnel $\stackrel{10}{\stackrel{1}{*}}$ The three triplet configurations, ${ }_{11}^{00} h_{T}^{2+}$, have the same energy as the singlet basis state ${ }_{11}^{00} h_{S}^{2+}$, namely $V_{B T}^{h h}-f$. They are fully decoupled.

Thus, the kinetic $h$ - $h$ exchange splitting arises in the 2-hole energy diagram, and the $e-h$ exchange splitting arises in the $X^{2+}$ energy diagram. Therefore, the transition spectrum, which is the difference between the two energy diagrams, shows separately both types of spin fine structure (Fig. 5(a) and (c)). On the right are the 2-hole spin patterns, and on the left are the the $X^{2+}$ spin patterns.

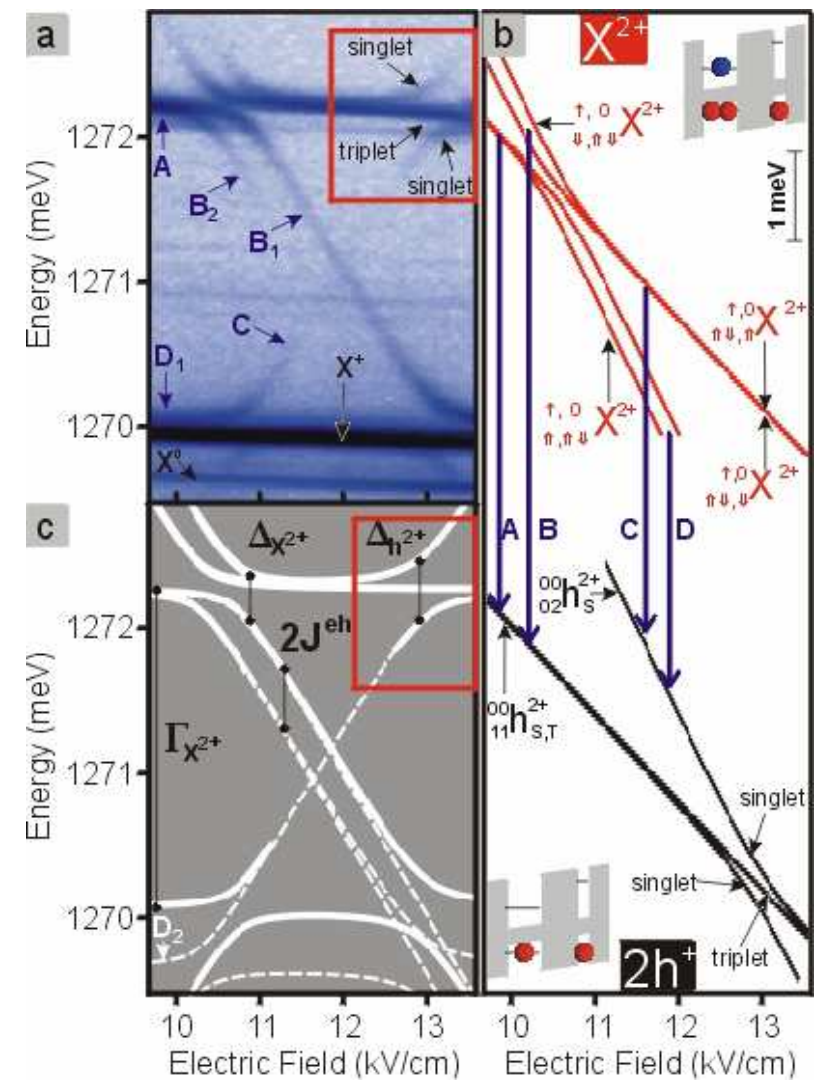

FIG. 5: (Color online) Doubly positively charged exciton $\left(X^{2+}\right)$ in a $\operatorname{QDM}\left(h_{B}=4 \mathrm{~nm}, h_{T}=2.5 \mathrm{~nm}, d=4 \mathrm{~nm}\right)$. (a) In the measured PL spectrum as a function of electric field an ' $\mathrm{x}$ '-shaped pattern is formed by the $X^{2+}$ transitions $\left(A \equiv \underline{2}_{1}^{10} X^{2+}, B \equiv \frac{10}{1} X^{2+}, C \equiv{ }_{21}^{10} X^{2+} \rightarrow{ }_{02}^{00} h^{2+}, D \equiv\right.$ $\left.{ }_{1}^{10} X^{2+}\right)$. (b) The calculated level diagram contains the states of the $X^{2+}$ (red lines) and the 2-hole states $\left(h^{2+}\right.$ ) (black lines). The calculation is done using a fit to four parameters $-t_{h}, J^{e h}, \Gamma_{X^{2+}} \stackrel{26}{ }$ and $p\left(\Delta_{h^{2+}}=2 \sqrt{2} t_{h^{+}}=410 \mu \mathrm{eV}\right.$, $\Delta_{X^{2+}}=2 t_{h^{+}}=310 \mu \mathrm{eV}, 2 J^{e h}=410 \mu \mathrm{eV}, \Gamma_{X^{2+}}=2.19$ $\mathrm{meV}, p=0.99 \mathrm{meV} /(\mathrm{kV} / \mathrm{cm}))$. Note that another 2-hole state in which both holes are in the bottom dot exists, but is not seen in the displayed energy range. The two different field dependences seen in the 2-hole and $X^{2+}$ states lead to the field-independent direct $(A$ and $D)$ and field-dependent indirect $(B$ and $C$ ) transitions. $B$ and $D$ are split by the $e-h$ exchange $J^{e h}$ of the optically excited state $\left(X^{2+}\right)$. The red box in (a) marks an area where the singlet-triplet splitting of the resonance of the 2-hole states in (b) is reproduced nicely by the PL signal. The anticrossing appears rotated because it occurs in the final state of the transitions $(A$ and $C$ ). (c) Calculated PL spectrum. The solid lines resemble the spectrum in (a). The dashed lines map transitions that are optically weak or forbidden by optical selection rules.

Note, some transitions are optically weak (e.g. $B_{2} \equiv$ $\stackrel{\uparrow}{\uparrow, 0}, \underline{\underline{1}} X^{2+}$ ) or forbidden by the optical selection rules (e.g. $D_{2} \equiv \underset{\uparrow}{\uparrow}, 0 \pi X^{2+}$ ) (white dashed lines in Fig. $5(\mathrm{c})$ ), but become visible in the vicinity of anticrossings. There they gain some oscillator strength from states with 
optically stronger transitions.

\section{INTERPLAY OF ELECTRON-HOLE AND KINETIC EXCHANGE: SINGLY CHARGED EXCITON}

With the $X^{+}$transitions we probe the QDM when it is charged with a single hole (see Fig. 6). In the $X^{+}$ states kinetic $h$ - $h$ exchange and $e-h$ exchange are both present and compete to determine the character of the spin state. In Fig. 6(a) the spectral pattern for the positive trion, $X^{+}$, is shown. This pattern can be readily understood using the energy state diagrams in Fig. 6(b) of both the trion and the hole that is left behind after recombination. The discovery of this overall " $\mathrm{x}$ "-pattern and its identification was made in Ref. 10. However, the spin fine structure was only partially interpreted. Now we are able to complete the description of the measured fine structure - at least at the level of the current experimental resolution. We focus our discussion on the anticrossing pattern in the box in Fig. 6(a), in which an apparent triplet transition wiggles as it passes through the resonance.

At electric fields away from the anticrossing region, intradot $e-h$ exchange determines the spin structure of the $X^{+}$. That is, as shown in the top of Fig. 6(b), $e-h$ exchange leads to a fine structure doublet with a splitting of $2 J^{e h}$, much like the intradot $X^{0}$ case. The higher energy component consists of the electron and one hole in the bottom dot with their spins antiparallel $\left(\begin{array}{l}\uparrow, 0 \\ \Downarrow, \Uparrow\end{array} X^{+}, \underset{\Downarrow, 0}{\uparrow, \Downarrow} X^{+}\right)$, while the lower energy component consists of parallel electron and hole spin in the bottom $\operatorname{dot}\left({ }_{\Uparrow, \Downarrow}^{\uparrow, 0} X^{+},{ }_{\Uparrow, \uparrow}^{\uparrow, 0} X^{+}\right) \underline{19,29}$ As the electric field is tuned through the $X^{+}$anticrossing region, tunnel coupling with the singlet $\underset{\uparrow \Downarrow, 0,0}{\uparrow, 0} X_{S}^{+}$state forces the spin states $\left(\underset{\Downarrow, \Uparrow}{\uparrow, 0} X^{+}\right.$, $\left.\stackrel{\uparrow, 0}{\Uparrow, \Downarrow} X^{+}\right)$to form a hole spin singlet-like state $\left(\underset{\Downarrow, \Uparrow}{\uparrow, 0} X_{S}^{+}\right)$and a hole spin triplet-like state $\left(\stackrel{\uparrow, 0}{\Downarrow, \Uparrow} X_{T}^{+}\right)$. This triplet would pass straight through the resonance (as with the 2-hole states) except that $e-h$ exchange continues to couple it to the singlets, causing it to shift continuously between the asymptotes determined by the $e-h$ exchange splitting outside the anticrossing region. Essentially, in passing through the anticrossing region (from right to left) the ${ }_{\Downarrow, 0}^{\uparrow, 0} X^{+}$state evolves continuously into the ${ }_{\Uparrow, \Downarrow}^{\uparrow, 0} X^{+}$state through this triplet-like state.

The Hamiltonian that describes this behavior of the basis states $|1\rangle={ }_{\Uparrow \Downarrow, 0}^{\uparrow, 0} X_{S}^{+},|2\rangle={ }_{\Downarrow, \Uparrow}^{\uparrow, 0} X^{+}$and $|3\rangle={ }_{\Uparrow, \Downarrow}^{\uparrow, 0} X^{+}$ is 29

$$
\widehat{H}_{\Uparrow \Downarrow}^{X^{+}}=\left(\begin{array}{ccc}
E_{B} & t_{h} & t_{h} \\
t_{h} & E_{B T}-f+J^{e h} & 0 \\
t_{h} & 0 & E_{B T}-f-J^{e h}
\end{array}\right) .
$$

The complete Hamiltonian for all six basis states, i.e. also including $\underset{0, \Uparrow \Downarrow}{\uparrow, 0} X_{S}^{+}, \stackrel{\uparrow, 0}{\Downarrow, \Downarrow} X^{+}$and $\stackrel{\Uparrow}{\uparrow, \uparrow}, 0^{\uparrow} X^{+}$, is given in ap-

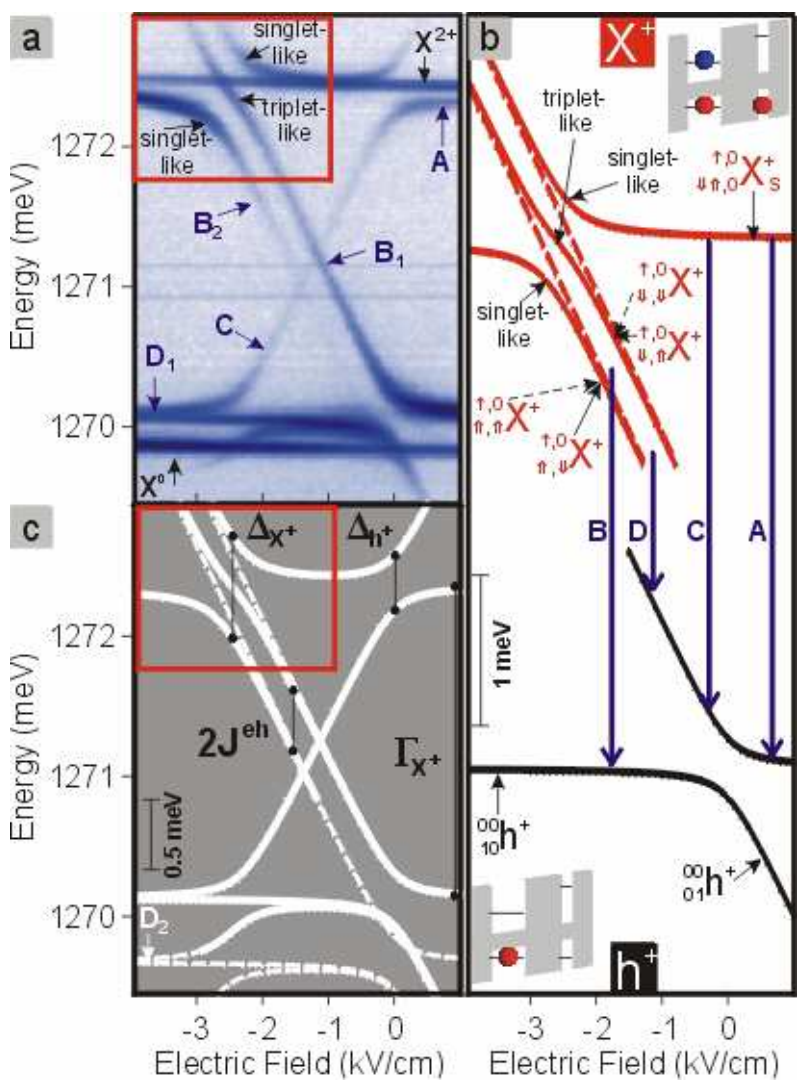

FIG. 6: (Color online) (a) In the measured PL spectrum as a function of electric field an ' $\mathrm{x}$ '-shaped pattern is formed by the $X^{+}$transitions $\left(A \equiv \underline{12}_{0}^{0} X^{+}, B \equiv \frac{10}{11}_{10} X^{+}, C \equiv{ }_{11}^{10} X^{+} \rightarrow\right.$ $\left.{ }_{01}^{00} h^{+}, D \equiv{ }_{11}^{10} X^{+}\right)$. (b) The calculated level diagram contains the states of the positive trion $\left(X^{+}\right)$(red lines) and the states of a single hole $\left(h^{+}\right)$(black lines) $\left(\Delta_{h^{+}}=2 t_{h^{+}}=390 \mu \mathrm{eV}\right.$, $\Delta_{X^{+}}=2 \sqrt{2 t_{h}^{2}+\left(J^{e h}\right)^{2}}=750 \mu \mathrm{eV}, 2 J^{e h}=430 \mu \mathrm{eV}, \Gamma_{X^{+}}=$ $\left.2.21 \mathrm{meV},{ }^{26} p=0.99 \mathrm{meV} /(\mathrm{kV} / \mathrm{cm})\right)$. Note that another $X^{+}$ state in which both holes are in the top dot exists, but is not seen in the displayed energy range. The two different field dependences seen in the $h^{+}$and $X^{+}$states lead to the fieldindependent direct $(A$ and $D)$ and field-dependent indirect $(B$ and $C$ ) transitions. $B$ and $D$ are split by the $e-h$ exchange $J^{e h}$ of the optically excited state $\left(X^{+}\right)$. The red box in (a) marks an area where the singlet-triplet mixing at the $X^{+}$ resonance in (b) is reproduced nicely by the PL signal. In the lower left corner of (a) the anticrossing is reproduced by the transitions $D$ and $C$ with different intensity ratios, but is partially covered by $\frac{10}{10} X^{0}$. (c) Calculated PL spectrum. The solid lines resemble the spectrum in (a). The dashed lines map transitions that are optically weak or forbidden by optical selection rules.

pendix B. With energy and field measured relative to the anticrossing of the hole states, $E_{B}$ is the energy of ${ }_{\Uparrow \Downarrow, 0}^{\uparrow, 0} X_{S}^{+}$and $E_{B T}=E_{X_{B}}-V_{B T}^{e h}-V_{B B}^{e h}+V_{B B}^{h h}-V_{B T}^{h h}$. Again not all transitions between the $X^{+}$states and the $h^{+}$states are observed in the measured PL spectrum, because they are optically weak (e.g. $\left.\frac{\uparrow}{\Downarrow, \underline{w}}, X^{+}\left(B_{1}\right)\right)$ or 
forbidden (e.g. $D_{2} \equiv \stackrel{\uparrow, 0}{\Uparrow, \Uparrow} X^{+}$) (Fig. 6(c), white dashed lines).

An alternative representation of the $X^{+}$as compared to Eq. (6) can be given in terms of the basis $|1\rangle=$ ${ }_{\uparrow \Downarrow \Downarrow, 0}^{\uparrow, 0} X_{S}^{+},|2\rangle={ }_{\uparrow, \Downarrow}^{\uparrow, 0} X_{S}^{+}$and $|3\rangle={ }_{\Uparrow, \Downarrow}^{\uparrow, 0} X_{T}^{+10}$. The transformation of Eq. (6) to this basis yields

$$
\widehat{H}_{\Uparrow \Downarrow}^{X^{+}}=\left(\begin{array}{ccc}
E_{B} & \sqrt{2} t_{h} & 0 \\
\sqrt{2} t_{h} & E_{B T}-f & J^{e h} \\
0 & J^{e h} & E_{B T}-f
\end{array}\right) .
$$

In this representation it becomes obvious that the $e-h$ exchange couples the triplet ${ }_{\uparrow, \Downarrow}^{\uparrow, 0} X_{T}^{+}$to the singlets.

Tunneling and $e-h$ exchange lead to a measured anticrossing energy $\Delta_{X^{+}}=2 \sqrt{2 t_{h}^{2}+\left(J^{e h}\right)^{2}}$. The remaining hole spin triplet states $\left({ }_{\Downarrow,, 0}^{\uparrow, 0} X^{+},{ }_{\uparrow, 0}^{\uparrow} X^{+}\right)$retain their character and pass unaffected through the coupling region as shown in Fig. 6(b) (red dashed lines), because Pauli blocking prevents the holes from tunneling. Thus, at the anticrossing point there is a kinetic exchange splitting between singlet- and triplet-like states, but $e-h$ exchange splits the degeneracy between the triplet states and leads to a mixing between the singlets and one of the triplets.

Interestingly, the singlet-triplet mixing observed here is similar to that found in transport studies $\frac{4,5}{\underline{5}}$ In that case hyperfine interactions, which are different in the two QDs, break the singlet-triplet symmetry of two electrons away from resonance. Here it is the $e-h$ exchange with the localized electron in the bottom QD, that breaks the singlet-triplet symmetry away from resonance. In both cases the strong tunneling term partially restores the singlet-triplet symmetry. However a residual mixing remains that results in the triplet "wiggling".

\section{CONCLUSIONS}

We have shown that the fine structure patterns measured in the optical spectra of QDMs are understood in detail in terms of the interplay between spin exchange, Pauli principle and tunneling in the limit of wide barriers and negligible direct interdot exchange. Our description applies equally well to electron tunneling and negatively charged QDMs. We note that we have also measured the neutral biexciton, which is found to have spin structure qualitatively similar to the $X^{2+}$ as expected $\stackrel{30}{=}$ Interesting but more subtle effects such as fine structure due to asymmetries (e.g. lateral displacement of the dots) have for the most part remained below the resolution of our measurements. In cases where the barrier is relatively thin $10,12,13$ such that wave function overlap becomes large, we expect additional interactions (such as direct interdot exchange) to become significant.

An important implication of these results is that exchange is effectively turned off (or on) when the
QDM is optically excited to specific spin states, thereby providing the opportunity for an ultrafast single qubit or 2-qubit operation. For example, the kinetic exchange interaction that splits the triplet and singlet states of the 2-holes, ${ }_{11}^{00} h^{2+}$, could be 'optically gated' for a well defined time by driving the QDM up and down through a ${ }_{21}^{10} X^{2+}$ state (i.e. a virtual $\frac{10}{2} 1 X^{2+}$ transition).

\section{APPENDIX A: ELECTRIC FIELD VALUES OF MOLECULAR RESONANCES}

Anticrossings are observed in the field dependent spectral map of a QDM when interdot transitions cross intradot transitions for a given charge state (see Fig. 1),$\underline{8,10}$ At the anticrossing, a hole (electron) becomes delocalized across both dots, forming bonding and antibonding molecular states. Different charge configurations become energetically degenerate (or resonant) at different applied electric fields because of different Coulomb interactions.

The relative position in electric field where the corresponding anticrossings occur in the spectrum depend on the Coulomb interactions between the involved charges. Figure 7 demonstrates this schematically for the cases when the QDM system is occupied by one hole only and when it is occupied by one hole and one electron. If we tune the ground state hole levels of the bottom and the top QD in resonance, the hole can be in either one of the dots while the electron remains localized in the bottom dot only. With only one hole no Coulomb interactions have to be considered and the electric field needed to tune the hole levels into resonance has to account only for the offset between the hole levels at zero applied field. Without loss of generality we set the field at which this resonance occurs to zero (Fig. 7(a)). With the additional electron in the bottom QD we have to account for the $e$ $h$ Coulomb interaction in both, intradot and interdot, charge configurations $-V_{B B}^{e h}$ and $V_{B T}^{e h}$. At resonance the hole has the same potential energy in both configurations (here we ignore the formation of bonding and antibonding states), and it follows that the corresponding electric field is proportional to the difference of the two Coulomb terms.

$$
F_{X^{0}}=\left(V_{B B}^{e h}-V_{B T}^{e h}\right) / p=\delta_{e h} / p
$$

$\delta_{e h}$ is the energy difference between the two charge configurations at the resonance of the bare hole (see Fig. 7(c)). Equivalently it is the energy which is required to move one hole from the bottom to the top QD while an electron resides in the bottom QD.

In Eq. (A1) we have neglected $e$-h exchange, $J^{e h}$. If we take it into account Eq. (A1) reads:

$$
F_{X^{0}}=\left(\delta_{e h} \pm J^{e h}\right) / p .
$$

The relative electric field of any other level resonance 

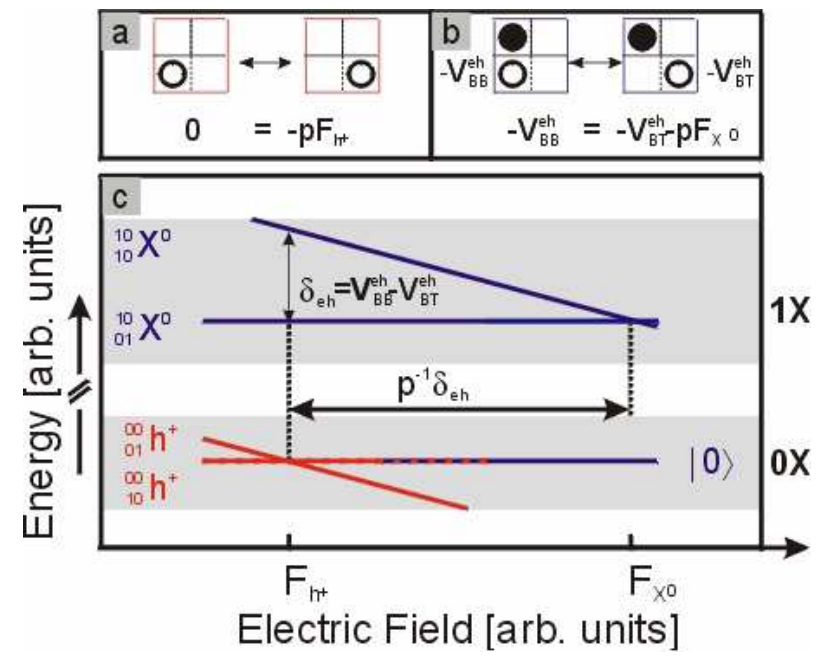

FIG. 7: (Color online)(a) Condition for a hole level resonance if the QDM is occupied by a hole only. (b) Condition for a hole level resonance if the QDM is occupied by a hole and an electron that is fixed to the bottom QD. (c) Geometrical interpretation of the separation between both resonances. can be found in analogy. For example, for the hole level resonance of the $X^{2+}$ we obtain ${ }^{31}$

$$
F_{X^{2+}}=\left(\delta_{e h}+V_{T T}^{h h}-V_{B B}^{h h} \pm J^{e h}\right) / p
$$

The fact that in Fig. 1 the molecular resonance of the $X^{2+}$ occurs at an electric field between the molecular resonances of the bare hole and the neutral exciton indicates that in this molecule $V_{T T}^{h h}$ is smaller than $V_{B B}^{h h}$.

\section{APPENDIX B: $6 \times 6$ HAMILTONIANS OF THE 2-HOLES \& THE POSITIVE TRION}

All three singlet and three triplet states of the two holes are described by a $6 \times 6$ Hamiltonian. In the basis $|1\rangle={ }_{0, \Uparrow \Downarrow}^{0,0} h_{S}^{2+},|2\rangle={ }_{\Uparrow, \Downarrow}^{0,0} h_{S}^{2+},|3\rangle={ }_{\Uparrow \Uparrow,, 0}^{0,0} h_{S}^{2+},|4\rangle={ }_{\Uparrow, \Uparrow}^{0,0} h_{T}^{2+}$, $|5\rangle={ }_{\Uparrow, \Downarrow}^{0,0} h_{T}^{2+}$ and $|6\rangle=\underset{\Downarrow, \Downarrow}{0,0} h_{T}^{2+}$ it is

$$
\widehat{H}^{h^{2+}}=\left(\begin{array}{cccccc}
V_{T T}^{h h}-2 f & \sqrt{2} t_{h} & 0 & 0 & 0 & 0 \\
\sqrt{2} t_{h} & V_{B T}^{h h}-f & \sqrt{2} t_{h} & 0 & 0 & 0 \\
0 & \sqrt{2} t_{h} & V_{B B}^{h h} & 0 & 0 & 0 \\
0 & 0 & 0 & V_{B T}^{h h}-f & 0 & 0 \\
0 & 0 & 0 & 0 & V_{B T}^{h h}-f & 0 \\
0 & 0 & 0 & 0 & 0 & V_{B T}^{h h}-f
\end{array}\right)
$$

In Eq. (6) we suppressed the basis states ${ }_{0, \uparrow \Downarrow}^{\uparrow, 0} X_{S}^{+}, \underset{\Downarrow, \Downarrow}{\uparrow, 0} X^{+}$and ${ }_{\Uparrow, \uparrow}^{\uparrow, 0} X^{+}$. The $6 \times 6$ Hamiltonian for the $X^{+}$in the basis $|1\rangle={ }_{0, \uparrow \Downarrow}^{\uparrow, 0} X_{S}^{+},|2\rangle=\underset{\uparrow \Downarrow, 0}{\uparrow, 0} X_{S}^{+},|3\rangle={ }_{\Downarrow, \uparrow}^{\uparrow, 0} X^{+},|4\rangle={ }_{\uparrow, \Downarrow}^{\uparrow, 0} X^{+},|5\rangle={ }_{\Downarrow, \Downarrow}^{\uparrow, 0} X^{+}$and $|6\rangle={ }_{\Uparrow, \uparrow}^{\uparrow, 0} X^{+}$is:

$$
\widehat{H}_{\Uparrow \Downarrow}^{X^{+}}=\left(\begin{array}{cccccc}
E_{T}-2 f & 0 & t_{h} & t_{h} & 0 & 0 \\
0 & E_{B} & t_{h} & t_{h} & 0 & 0 \\
t_{h} & t_{h} & E_{B T}-f+J^{e h} & 0 & 0 & 0 \\
t_{h} & t_{h} & 0 & E_{B T}-f-J^{e h} & 0 & 0 \\
0 & 0 & 0 & 0 & E_{B T}-f+J^{e h} & 0 \\
0 & 0 & 0 & 0 & 0 & E_{B T}-f-J^{e h}
\end{array}\right),
$$

where $E_{T}=E_{B}+2\left(V_{B B}^{e h}-V_{B T}^{e h}\right)+V_{T T}^{h h}-V_{B B}^{h h}$. In terms of the alternative basis, $|1\rangle=\underset{0, \uparrow \Downarrow}{\uparrow, 0} X_{S}^{+},|2\rangle=\stackrel{\uparrow, 0}{, 0} X_{S}^{+},|3\rangle=$ $\stackrel{\uparrow, 0}{\Uparrow, \Downarrow} X_{S}^{+},|4\rangle={ }_{\uparrow, \Downarrow}^{\uparrow, 0} X_{T}^{+},|5\rangle=\underset{\Downarrow, \Downarrow}{\uparrow, 0} X^{+}$and $|6\rangle={ }_{\uparrow, \uparrow}^{\uparrow, 0} X^{+}$the Hamiltonian of the $X^{+}$reads:

$$
\widehat{H}_{\Uparrow \Downarrow}^{X^{+}}=\left(\begin{array}{cccccc}
E_{T}-2 f & 0 & \sqrt{2} t_{h} & 0 & 0 & 0 \\
0 & E_{B} & \sqrt{2} t_{h} & 0 & 0 & 0 \\
\sqrt{2} t_{h} & \sqrt{2} t_{h} & E_{B T}-f & J^{e h} & 0 & 0 \\
0 & 0 & J^{e h} & E_{B T}-f & 0 & 0 \\
0 & 0 & 0 & 0 & E_{B T}-f+J^{e h} & 0 \\
0 & 0 & 0 & 0 & 0 & E_{B T}-f-J^{e h}
\end{array}\right) .
$$




\section{ACKNOWLEDGMENTS}

We would like to acknowledge the financial support by NSA/ARO, CRDF, RFBR, RSSF, and ONR. M.F.D.,
I.V.P and E.A.S, are NRC/NRL Research Associates.
* Electronic address: scheibner@bloch.nrl.navy.mil

1 D. Loss, D.P. DiVincenzo, Phys. Rev. A 57, 120 (1998).

2 D. P. DiVincenzo, D. Bacon, J. Kempe, G. Burkard and K. B. Whaley, Nature 408, 339 (2000).

3 D. Awschalom, D. Loss and N. Samarth (eds.), Semiconductor Spintronics and Quantum Computation (Springer, 2002), ISBN 3540421769.

4 J. R. Petta, A. C. Johnson, J. M. Taylor, E. A. Laird, A. Yacoby, M. D. Lukin, C. M. Marcus, M. P. Hanson, and A. C. Gossard, Science 309, 2180 (2005).

${ }^{5}$ F. H. L. Koppens, J. A. Folk, J. M. Elzerman, R. Hanson, L. H. Willems van Beverem, I. T. Vink, H. P. Tranitz, W. Wegscheider, L. P. Kouwenhoven, and L. M. K. Vandersypen, Science 309, 1346 (2005).

${ }^{6}$ D. Gammon, E. S. Snow, B. V. Shanabrook, D. S. Katzer, and D. Park, Phys. Rev. Lett. 76, 3005 (1996).

7 B. Urbaszek, R. J.Warburton, K. Karrai, B. D. Gerardot, P.M. Petroff, and J.M. Garcia, Phys. Rev. Lett. 90, 247403 (2003).

8 H. J. Krenner, M. Sabathil, E. C. Clark, A. Kress, D. Schuh, M. Bichler, G. Abstreiter, and J. J. Finley, Phys. Rev. Lett. 94, 057402 (2005).

9 G. Ortner, M. Bayer, Y. Lyanda-Geller, T. L. Reinecke, A. Kress, J. P. Reithmaier, and A. Forchel, Phys. Rev. Lett. 94, 157401 (2005).

10 E. A. Stinaff, M. Scheibner, A. S. Bracker, I. V. Ponomarev, V. L. Korenev, M. E. Ware, M. F. Doty, T. L. Reinecke, D. Gammon, Science 311, 636 (2006).

11 T. Nakaoka, E. C. Clark, H. J. Krenner, M. Sabathil, M. Bichler, Y. Arakawa, G. Abstreiter, and J. J. Finley, Phys. Rev. B 74, 121305 (2006).

12 M. F. Doty, M. Scheibner, I.V. Ponomarev, E. A. Stinaff, A. S. Bracker, V. L. Korenev, T. L. Reinecke, and D. Gammon, Phys. Rev. Lett. 97, 197202 (2006).

13 A. S. Bracker, M. Scheibner, M. F. Doty, E. A. Stinaff, I. V. Ponomarev, J. C. Kim, L. J. Whitman, T. L. Reinecke, and D. Gammon, Appl. Phys. Lett. 89, 233110 (2006).

14 H. J. Krenner, E. C. Clark, T. Nakaoka, M. Bichler, C. Scheurer, G. Abstreiter, and J. J. Finley, Phys. Rev. Lett. 97, 076403 (2006).

15 I. V. Ponomarev, M. Scheibner, E. A. Stinaff, A. S. Bracker, M. F. Doty, S. C. Badescu, M. E. Ware, V. L. Korenev, T. L. Reinecke, D. Gammon, phys. stat. sol. (b) 243, 3869 (2006).

16 R. J. Warburton, C. Schäflein, D. Haft, F. Bickel, A. Lorke, K. Karrai, J. M. Garcia, W. Schoenfeld and P. M. Petroff, Nature 405, 926 (2000).

17 M. E. Ware, E. A. Stinaff, D. Gammon, M. F. Doty, A. S. Bracker, D. Gershoni, V. L. Korenev, S. C. Badescu, Y. Lyanda-Geller, and T. L. Reinecke, Phys. Rev. Lett. 95, 177403 (2005).

18 K. V. Kavokin, Phys. Rev. B 69, 075302 (2004).

$19 \uparrow, 0, X_{S(T)}^{+} \equiv \frac{1}{2}\left|B_{1}^{h} T_{2}^{h} \pm B_{2}^{h} T_{1}^{h}\right\rangle\left|\Downarrow_{1} \Uparrow_{2} \mp \Downarrow_{2} \Uparrow_{1}\right\rangle\left|B^{e} \uparrow\right\rangle$, where $B^{h(e)}$ and $T^{h}$ denote the primary location of the orthonormal orbital wavefunctions of the electron and the two holes.

20 I. V. Ponomarev, M. Scheibner, A. S. Bracker, M. F. Doty, D. Gammon and T. L. Reinecke, (unpublished).

21 J. G. Tischler, A. S. Bracker, D. Gammon, and D. Park, Phys. Rev. B 66, 081310(R) (2002).

22 M. Bayer, G. Ortner, O. Stern, A. Kuther, A. A. Gorbunov, A. Forchel, P. Hawrylak, S. Fafard, K. Hinzer, T. L. Reinecke, S. N. Walck, J. P. Reithmaier, F. Klopf, and F. Schäfer, Phys. Rev. B 65, 195315 (2002).

23 M. Bayer, O. Stern, A. Kuther, A. Forchel, Phys. Rev. B 61, 7273 (2000).

24 For wide barriers contributions to the tunneling rate due to the presence of additional charges are negligible ${ }^{10}$.

25 G. Burkard, G. Seelig and D. Loss, Phys. Rev. B 62, 2581 (2000)

${ }^{26} \Gamma_{X^{2+}}=\Gamma_{X^{+}}=V_{B T}^{e h}-V_{B B}^{e h}+V_{B B}^{h h}-V_{B T}^{h h}-J^{e h}$.

27 The ${ }_{20}^{00} h^{2+}$ state is at higher energy and is ignored here.

28 P. Fazekas, Lecture Notes on Electron Correlation and Magnetism (World Scientific, Singapore, 1999).

29 We define: $\underset{\Downarrow, \uparrow}{\uparrow, 0} X^{+}=\frac{1}{\sqrt{2}}\left(\underset{\Uparrow, \Downarrow}{\uparrow, 0} X_{S}^{+}+\underset{\Uparrow, \Downarrow}{\uparrow, 0} X_{T}^{+}\right)$and $\underset{\Uparrow, \Downarrow}{\uparrow, 0} X^{+}=$ $\frac{1}{\sqrt{2}}\left({ }_{\uparrow, \Downarrow}^{\uparrow, 0} X_{S}^{+}-{ }_{\Uparrow, \Downarrow}^{\uparrow, 0} X_{T}^{+}\right)$.

30 M. Scheibner, M. F. Doty, I. V. Ponomarev, A. S. Bracker, E. A. Stinaff, T. L. Reinecke, C. S. Hellberg and D. Gammon, arXiv:0705.1205 (unpublished).

31 M. Scheibner, A. S. Bracker, E. A. Stinaff, M. F. Doty, D. Gammon, I. V. Ponomarev, T. L. Reinecke, and V. L. Korenev, in PHYSICS OF SEMICONDUCTORS: 28th International Conference on the Physics of Semiconductors, edited by W. Jantsch, J. Kepler and F. Schaffler (American Institute of Physics, 2007), vol. 893, p. 871. 